\title{
Electrostatically driven synthetic microjet arrays as a propulsion method for micro flight
}

\section{Part II: microfabrication and initial characterization}

Received: 29 October 2004 / Accepted: 17 December 2004/Published online: 26 July 2005

(C) Springer-Verlag 2005

\begin{abstract}
A propulsion system based on acoustic streaming generated by Helmholtz resonators is presented. High frequency $(>60 \mathrm{kHz})$ electrostatically driven micromachined Helmholtz resonators constitute the basic unit of the system. Microjets produced at the exit of these resonators can be combined to form a distributed propulsion system. A high yield $(>85 \%)$ fabrication process is introduced for fabrication of individual as well as arrays of resonators. The fabrication results for ten different designs are presented. About 1000 resonators of similar design cover the surface of a 4 -in. wafer, effectively converting it to a distributed propulsion system. A number of characterization methods such as monitoring the harmonics of the drive current, laser interferometry, hot-wire anemometry, acoustic spectrum measurement and video particle imaging are used to determine the structural and fluidic behavior of different resonator designs. Collapse and recovery times of the diaphragm in the electrostatic actuator of the resonator are characterized and reduced to less than $10 \mu$ s by optimizing the perforation design. The occurrence of acoustic streaming in the micron-scale is verified via video particle imaging. The jet streams produced with
\end{abstract}

\footnotetext{
B. A. Parviz ( $\square)$

Department of Electrical Engineering,

University of Washington, Campus Box 352500 ,

Seattle, WA 98195, USA

E-mail: babak@ee.washington.edu

Tel.: + 1-206-6164038

Fax: + 1-206-5433842

K. Najafi

Department of Electrical Engineering and Computer Science,

Center for Wireless Integrated Micro Systems,

University of Michigan, Ann Arbor,

MI 48109, USA

M. O. Muller · L. P. Bernal · P. D. Washabaugh

Aerospace Engineering Department,

University of Michigan, Ann Arbor,

MI 48109, USA
}

pulse drive at low frequencies $(\sim 1 \mathrm{kHz})$ are spatially profiled and jet velocities exceeding $1 \mathrm{~m} / \mathrm{s}$ are measured at the exit of the resonators. It has been verified that the resonance frequencies of the device at 50 and $175 \mathrm{kHz}$ can be closely predicted by modeling.

\section{Introduction}

Jet generation in micro scale through acoustic streaming has been previously demonstrated and applied for jet vectoring and cooling of electronic circuits (Coe et al. 1994, 1995). These miniature jets can also be used to form a propulsion system. In (Parviz et al. 2005) our group presented the theoretical framework for design of micro jets based on acoustic streaming and Helmholtz resonance. In this method, oscillatory motion of an electrostatically actuated diaphragm generates an acoustic field inside the cavity of a Helmholtz resonator. The initial flow velocities are amplified by the resonator structure and create a jet stream upon exiting the resonator due to acoustic streaming. The equations introduced in (Parviz et al. 2005) are used in this paper to design micro Helmholtz resonators that form the unit cell for a micro propulsion system. Acoustic streaming and jet formation occur at the exit of these resonators and an array of them can be used directly for thrust generation.

In this paper we present a microfabrication process for making the Helmholtz resonator units. This highyield process has allowed us to fabricate large arrays of Helmholtz resonators covering the entire surface of a 4in. wafer. A high-frequency electrostatic actuator constitutes the driving force for the acoustic field inside the Helmholtz resonator cavity. Through an extensive study of the behavior of these actuators and varying their design parameters, we aim to improve the efficiency of the jet generation system. 


\section{Resonator description}

Figure 1 shows a schematic of a Helmholtz resonator. The resonator consists of a cavity made in Pyrex 7740 glass and a top silicon layer housing the electrostatic actuator and the resonator throats. Two configurations for throat geometry are considered with two or eight nozzles for each resonator. The electrostatic actuator is made of a conductive diaphragm separated from a perforated back-plate by the actuator gap. Application of a voltage between the two creates an attraction force that deflects the diaphragm. If the force is large enough the diaphragm completely collapses against the back plate and maximum volume displacement is achieved (collapse). When the voltage is removed, the diaphragm returns to the initial position (recovery). By application of an alternating voltage to the actuator, oscillatory motion of the diaphragm is achieved.

Critical parameters in the design of the electrostatic actuator are the diaphragm size, actuator gap, diaphragm residual stress, back-plate thickness and backplate perforation design. Back-plate perforation design determines to what extent air damping limits the high frequency motion of the diaphragm. Table 1 outlines the specifications for a sample resonator design (design\#1). In order to investigate the effect of various device parameters on performance, a number of devices were fabricated with different parameters. The main variables were the number and size of exit throats and the actuator perforation design. All devices had similar diaphragm, actuator gap and chamber dimensions. Table 2 details the specifications and calculated performances for each design. In this table the hole size and spacing specify the actuator back-plate perforation design. The non-uniform hole distribution in design\#5 was designed to match the collapse pattern of the diaphragm against

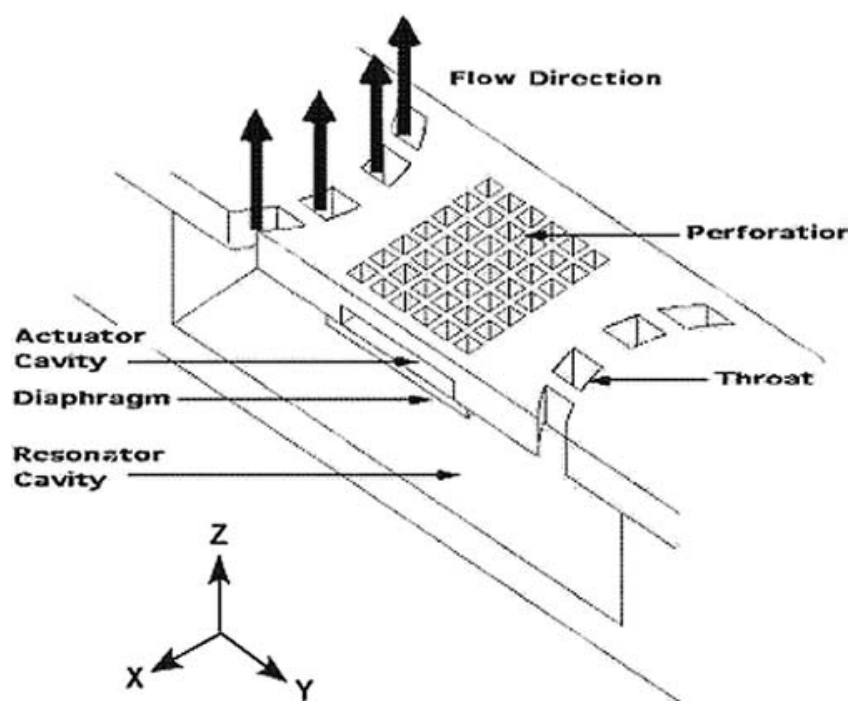

Fig. 1 Schematic of a Helmholtz resonator and an electrostatic actuator (not to scale) the back-plate. Note that the throat length was kept constant at $15 \mu \mathrm{m}$ for all designs. To reinforce the fabricated structure, in designs $\# 2-\# 9$, horizontal bars were placed across the throats effectively dividing them to a few smaller ones.

\section{The microfabrication process}

A two-wafer microfabrication process was developed to make the high frequency resonators and form the distributed propulsion system (Parviz et al. 2000). Figure 2 shows the simplified fabrication process flow. The process started with a $16 \mathrm{~h}$ solid-source boron diffusion step that formed a $15 \mu \mathrm{m}$ deep heavily doped region at the surface of the wafer. The actuator cavity was etched with a time-multiplexed deep etcher (Fig. 2a). It was filled with $0.2 \mu \mathrm{m}$ of silicon nitride and $3 \mu \mathrm{m}$ of low stress polysilicon deposited via Chemical Vapor Depositoin (CVD). The polysilicon was the sacrificial layer filling the actuator gap and was removed at the end of the process by wet etching. A $0.2 \mu \mathrm{m}$ silicon nitride layer was deposited to prevent boron diffusion from the highly doped region to the sacrificial polysilicon through the process. The process was continued by a Chemical Mechanical Polishing (CMP) step (Fig. 2b). This was done to reduce the step height on the wafer from $3.2 \mu \mathrm{m}$ to less than $0.1 \mu \mathrm{m}$ and prepare the surface for deposition of the composite diaphragm. Reduction of the step height was essential for successful fabrication of the diaphragm. The diaphragm was designed under high residual tensile stress and if it were not properly anchored at the edges, it could easily collapse or crack after release. The diaphragm formation started with deposition of $0.19 \mu \mathrm{m}$ of CVD silicon nitride and $0.31 \mu \mathrm{m}$ of CVD silicon dioxide. Then $0.8 \mu \mathrm{m}$ of low-stress poly silicon was deposited and doped with boron. This doping step was performed in order to render the diaphragm

Table 1 Design\#1 specifications

\begin{tabular}{ll}
\hline Parameter & Value \\
\hline $\begin{array}{l}\text { Diaphragm dimensions } \\
\text { Diaphragm composition }\end{array}$ & $\begin{array}{l}1200 \times 1200 \times 1.8 \mu \mathrm{m} \\
0.62 \mu \mathrm{m} \text { oxide; } 0.38 \mu \mathrm{m} \\
\text { nitride; } 0.8 \mu \mathrm{m} \text { poly } \\
+79.44 \mathrm{Mpa}\end{array}$ \\
$\begin{array}{l}\text { Diaphragm residual stress } \\
\begin{array}{l}\text { Diaphragm resonance } \\
\text { frequency }\end{array}\end{array}$ & $\begin{array}{l}104.7 \mathrm{KHz} \\
\text { Pressure Req. for } 1.5 \mu \mathrm{m} \\
\text { deflection }\end{array}$ \\
$\begin{array}{l}\text { Diaphragm DC collapse } \\
\text { voltage }\end{array}$ & $2046 \mathrm{~Pa}$ \\
$\begin{array}{l}\text { Electrostatic actuator gap } \\
\text { Chamber dimensions }\end{array}$ & $52.5 \mathrm{~V}$ \\
$\begin{array}{l}\text { Throat dimensions } \\
\text { Helmholtz resonance } \\
\text { frequency }\end{array}$ & $3 \mu \mathrm{m}$ \\
$\begin{array}{l}\text { \# Of throats } \\
\text { Device operating voltage at res. } \\
\text { Maximum power consumption }\end{array}$ & $1730(\mathrm{effective}) \times 1350 \times 35 \mu \mathrm{m}$ \\
\hline
\end{tabular}


Table 2 Design specifications and calculated performances

\begin{tabular}{|c|c|c|c|c|c|c|c|c|c|c|}
\hline Design\# & 1 & 2 & 3 & 4 & 5 & 6 & 7 & 8 & 9 & 10 \\
\hline Hole size $(\mu \mathrm{m})$ & 5 & 15 & 15 & 13 & $\mathrm{U}^{a}$ & 5 & 5 & 5 & 5 & 5 \\
\hline Spacing $(\mu \mathrm{m})$ & 5 & 15 & 15 & 5 & $\mathrm{U}^{a}$ & 5 & 5 & 5 & 5 & 5 \\
\hline Bar on throat & No & Yes & Yes & Yes & Yes & Yes & Yes & Yes & Yes & No \\
\hline No. of Throats & 2 & 8 & 8 & 8 & 8 & 8 & 8 & 8 & 8 & 2 \\
\hline Throat width $(\mu \mathrm{m})$ & 1400 & 330 & 330 & 330 & 330 & 330 & 330 & 330 & 330 & 1400 \\
\hline Res. Freq. (kHz) & 59.1 & 60.9 & 64.1 & 60.9 & 60.9 & 60.9 & 62.53 & 63.0 & 63.36 & 62.57 \\
\hline${ }^{b}$ Max flow length $(\mu \mathrm{m})$ & 82 & 90 & 37 & 90 & 90 & 90 & 67 & 60 & 53 & 53 \\
\hline${ }^{b}$ Max Thrust $(\mu \mathrm{N})$ & 3.33 & 3.15 & 2.40 & 3.15 & 3.15 & 3.15 & 3.52 & 3.31 & 3.08 & 3.25 \\
\hline
\end{tabular}

${ }^{a}$ Non-uniform hole distribution ${ }^{b}$ Calculated at resonance with $0.7 \mu \mathrm{m}$ diaphragm displacement amplitude

conductive (Fig. 2c). The polysilicon was etched by Reactive Ion Etching (RIE) down to the dielectric layer and then $0.31 \mu \mathrm{m}$ of silicon dioxide and $0.19 \mu \mathrm{m}$ of silicon nitride was deposited to cap the polysilicon and complete the diaphragm deposition (Fig. 2d). Contacts were opened in the dielectric by RIE and subsequently a metalization/lift-off step formed the gold electrical connectors (Fig. 2e). This completed the process on the silicon wafer. The second wafer was made of Pyrex 7740 glass and contained the resonator chambers. It also contained shallow trenches to allow the gold connector to traverse the length of the device, and via holes for electrical connection. The process on the glass wafer started with the sputtering of an etch mask $(\mathrm{Cr} / \mathrm{Au})$ and subsequent patterning of it by photoresist and wet etching of the metals. This metal mask was used for etching of the glass in an HF-nitric solution. After formation of the shallow trenches, the metal mask was removed and a similar procedure was repeated to form the resonance cavity. Note that the depth of the shallow trenches should be minimized to lower the leakage rate between the adjacent resonators since these trenches provide a path for the flow to move between adjacent resonators. The depth of the resonance chamber was $\sim 35 \mu \mathrm{m}$. We found that the undercut can vary widely depending on the glass surface quality and grain boundaries. In order to access the metal pads on the silicon wafer after bonding, via holes were drilled in the glass wafer with a $500 \mu \mathrm{m}$ drill bit.

After the individual processes on the glass and silicon wafer were finished, the wafers were anodically bonded. Anodic bonding is done customarily at temperatures
Fig. 2 The resonator fabrication process (not to scale)
A

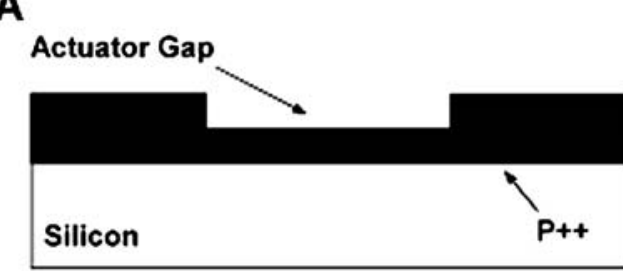

B

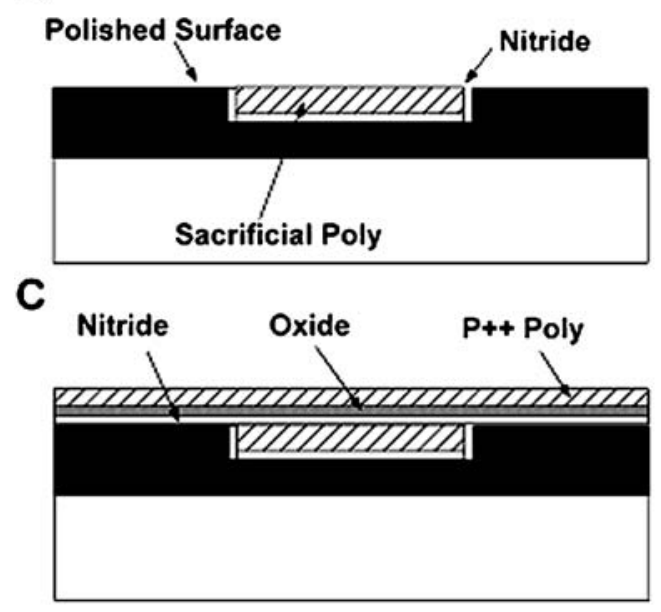

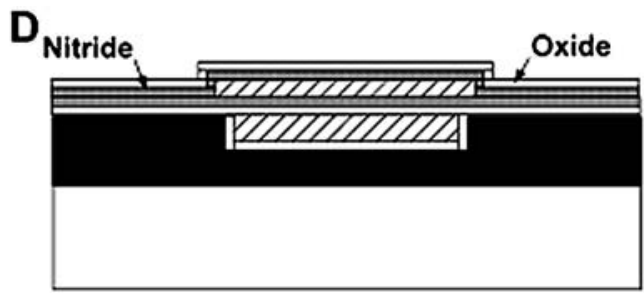

E

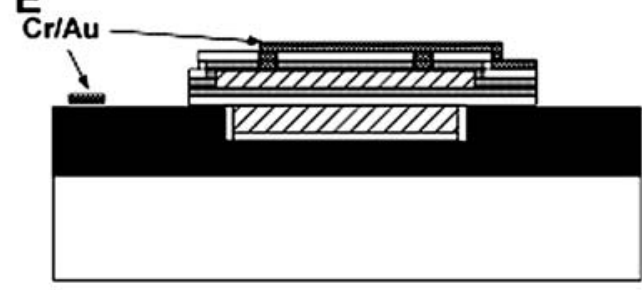

F

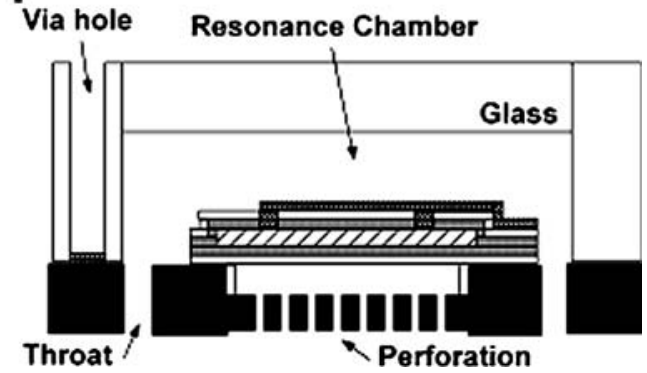


around $400^{\circ} \mathrm{C}$ (Wolffenbuttle and Wise 1994); however, by bonding at this temperature, sever degradation of the $\mathrm{Cr} / \mathrm{Au}$ connector lines on silicon was noticed. This was due to the diffusion of gold through the thin chromium layer and formation of the eutectic phase at the gold/ silicon interface. In order to prevent the degradation, the bonding was performed at temperatures as low as $300^{\circ} \mathrm{C}$ with satisfactory results. In order to improve the bonding surface a pre-bond Ethylene Diamine Pyrocatecol (EDP) etch can be utilized. Since the surface of the silicon wafer in the bonding areas is heavily boron doped, by a short exposure to EDP, most of the surface roughness can be smoothened without loss of the structural silicon. After bonding the wafer was etched in EDP to remove the excess silicon from the structure. This etch started from the backside of the silicon wafer and proceeded until the etch front met the heavily boron doped region in the device. This region effectively stopped the etching and the result was a mirror-finished surface made of $15 \mu \mathrm{m}$ of boron-doped silicon bonded to a glass substrate. The processing continued with a lithography step on this surface to define the exit throats and the perforation holes. After the lithography, these areas were etched by a time-multiplexed deep silicon etcher to the depth of $15 \mu \mathrm{m}$. This defined the perforation holes in the back-plate and the throats. At this point the $0.2 \mu \mathrm{m}$ CVD silicon nitride layer which was used as a diffusion barrier was exposed. This layer was etched by RIE in order to provide an access route to the sacrificial polysilicon inside the actuator cavity. The last step of the microfabrication process was the release of the actuator diaphragm. This was accomplished by a short EDP etch to remove the sacrificial polysilicon (Fig. 2f). At the end, the connector lines were attached using a combination of conductive and vacuum epoxies.

\section{Fabrication results}

Figure 3 shows a picture of a complete fabricated 4-in. wafer next to a US Quarter coin. The surface of the wafer was divided to four quadrants. Each quadrant was configured to form a micro acoustic thruster with 70 ejectors. The ejector shrouds were not fabricated on this wafer; however, the resonators were placed around an opening to facilitate future hybrid fabrication of the ejectors on the propulsion system. The inset shows six resonators around an ejector opening. To take this SEM image, the silicon section was detached from the glass substrate. Throats and connector lines are visible in the picture. Signal distribution is achieved by gold connector lines and in part by the conductive diaphragms. This distributed system is less prone to failure due to local disconnection of the connectors. The second inset shows a close-up of damping reduction holes on the back-plate etched in silicon. These were from design\#5 as detailed in Table 2. In the center region the holes were $13 \mu \mathrm{m}$ wide with $5 \mu \mathrm{m}$ spacing. The width of the holes gradually decreased to $6 \mu \mathrm{m}$ at the edge of the diaphragm area. This pattern matched the collapse pattern of the diaphragm against the back-plate. The 148 devices were tested on a quadrant of a fabricated wafer to measure the fabrication yield. The fabrication yield was higher than $85 \%$ with most of the non-functional devices located at the wafer edge far from the center.

\section{Experimental study of the fabricated devices}

A range of test and characterizations were performed on the fabricated devices in order to measure the basic fluidic and structural behavior of the system and also verify the models developed in (Parviz et al. 2005). Five categories of tests were performed on the resonators. These were electrical, acoustic, laser interferometry, video particle imaging and hot-wire anemometry. In total more than 300 devices were tested to collect the data in this section. Performing measurements on a miniature jet system proved to be a challenging task. Many of the traditional tools developed for aerodynamic test and characterization of larger scale systems were not applicable in this case. Although there were fabricated samples available in many cases, the limitation of the measurement set-ups impeded further data collection. The following sections detail the measurement methods and collected data from various designs. These are compared with the calculated values where applicable in order to investigate the validity of the models in (Parviz et al. 2005).

\subsection{Electrical characterization}

To measure the resonance frequencies of the system, harmonics of the driving current to the device were monitored. The resonance frequency of the device can be found by plotting the ratio of the third to the first harmonic (Parviz et al 2001; Muller et al. 2000). Figure 4 shows the first resonance point of a design\#1 device. The peak of this figure is at $26 \mathrm{kHz}$ which translates to $26 \mathrm{kHz} \times 2=52 \mathrm{kHz}$ for the first resonance frequency of the system. Multiplication by two is a result of frequency doubling by the electrostatic actuator. At $0.006 \mathrm{~atm}$, the first resonance point was measured at $56 \mathrm{kHz}$ and the second resonance point at $190 \mathrm{kHz}$. This showed a remarkable agreement with the theoretical predictions made using equations of reference (Parviz et al. 2005) for the device.

\subsection{Laser interferometry}

Laser interferometry is a powerful tool for characterization of a dynamic system with small amplitudes of motion. A YAG laser was directed at the resonator diaphragm surface. A high-speed digital camera imaged the fringe pattern formed by the primary beam and the reflected beam from the diaphragm. The camera was 
Fig. 3 A finished array of Helmholtz resonators next to a US Quarter coin. The wafer contains about 1000 resonators configured in four individually addressable quadrants. The insets show six resonators surrounding an ejector opening, and details of the non-uniform hole distribution on the actuator back-plate for design\#5

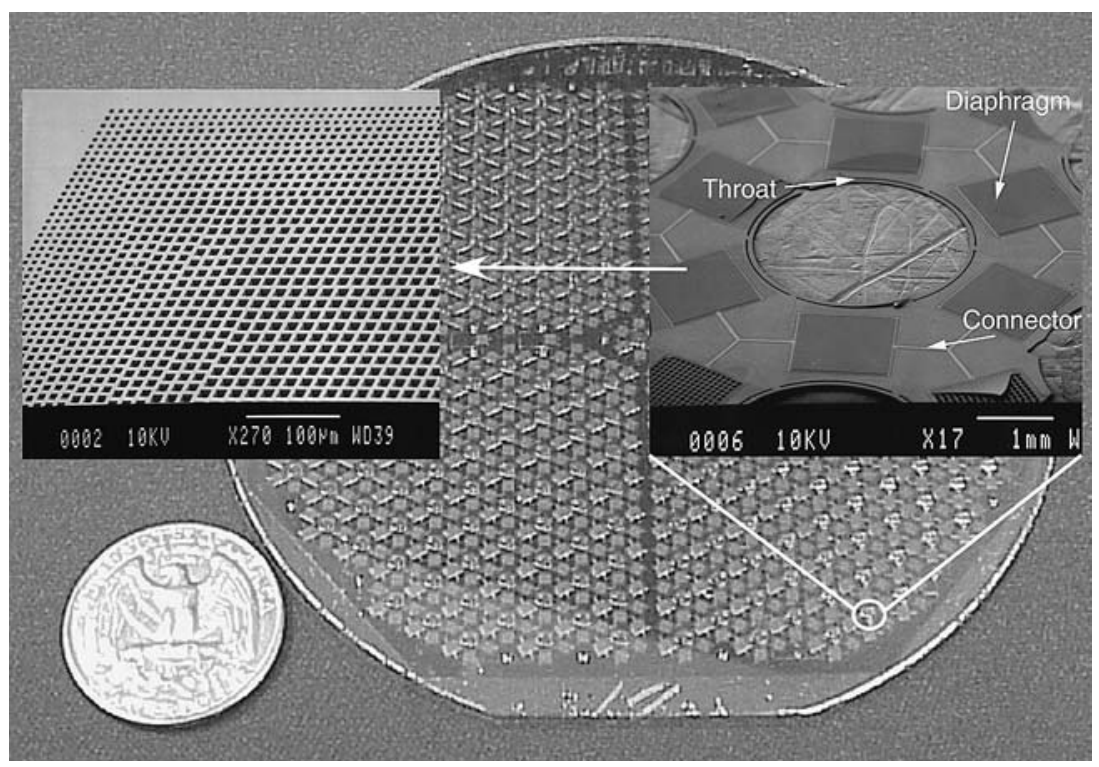

triggered by the input signal to the resonator. The camera was controlled by a personal computer and the shutter open interval and image quality could be modified through the control software. The measured average collapse voltage for the diaphragms was $60 \mathrm{~V}$, which was higher than the $52.5 \mathrm{~V}$ predicted by modeling. This collapse voltage corresponds to $+20 \mathrm{MPa}$ residual stress in the doped polysilicon layer. The volume displacement due to diaphragm motion can also be characterized dynamically with the interferometric measurement. A design\#1 sample was actuated by application of a sine wave with the amplitude of $65 \mathrm{~V}$ at $30 \mathrm{kHz}$. The diaphragm under this operating condition never completely released during the full actuation cycle and also showed significant asymmetry in the negative and positive cycles. We calculated the total volume under the diaphragm for each state by assigning $0.244 \mu \mathrm{m}$

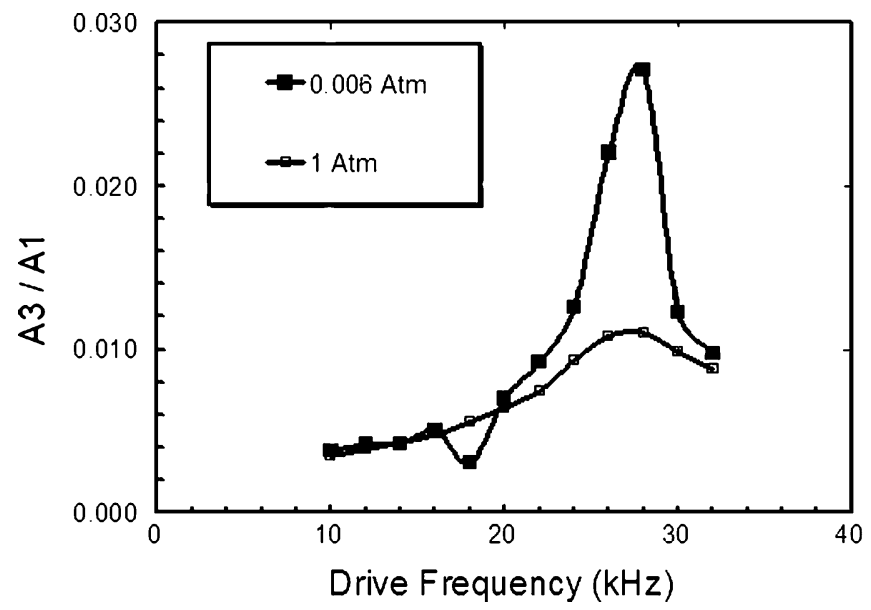

Fig. 4 Measured ratio of third and first harmonics of the drive current versus driving voltage frequency (half the diaphragm motion frequency). The experiment was performed at ambient and low pressure displacement for each fringe (in accordance to the laser wavelength) and dividing each image to areas of similar displacement. In high frequency operations, before the diaphragm reached the zero deflection point a new cycle started and thus the diaphragm started to move back again. This produced a new dynamic equilibrium point for the oscillations of the diaphragm. This point changed with changing the amplitude of the applied signal.

If the volume displacement in each state is divided by the area of the diaphragm, an equivalent piston motion displacement can be derived for the diaphragm. This piston motion is always in the same direction, increasing the volume displaced under the diaphragm. Table 2 lists the calculated performance based on this displacement. Note that this piston displacement is equivalent to $0.7 \mu \mathrm{m}$ center deflection for the diaphragm in Table 2 due to curvature of the diaphragm.

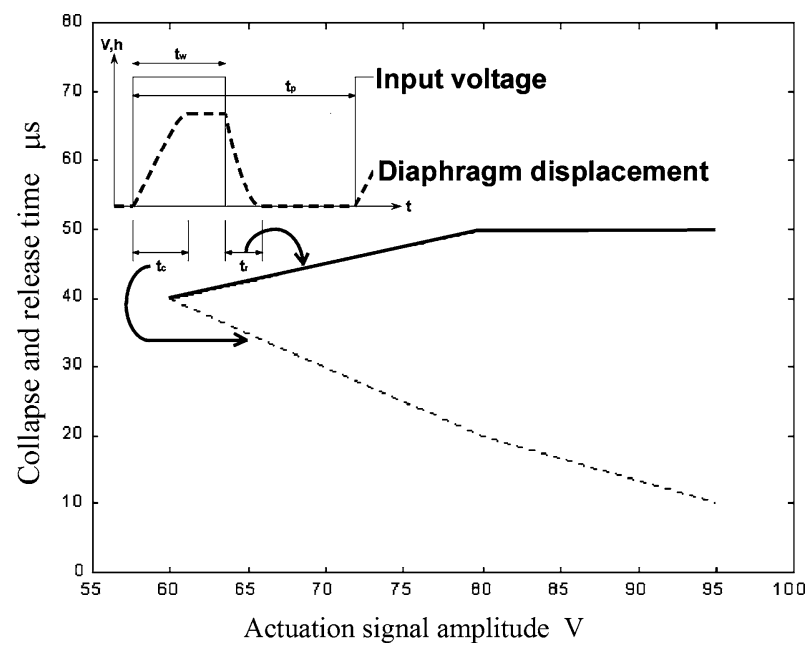

Fig. 5 Measured diaphragm pulse response. The inset shows the definition of rise time and the collapse time. The applied pulse was $60 \mu$ s long 
In order to investigate the effect of charging on the device operation, a series of tests were performed using a square pulse shape. The pulse width $\left(t_{w}\right)$, pulse amplitude $\left(V_{A}\right)$, and polarity were varied and collapse time $\left(t_{c}\right)$ and release times $\left(t_{r}\right)$ for a design\#1 diaphragm were measured (Fig. 5). The pulse period $\left(t_{p}\right)$ was chosen at $2000 \mu$ s to reduce the effect of accumulative charging and provide enough time for dissipation. The release of the diaphragm showed an over-damped behavior. After the end of the pulse, the diaphragm returned to the released state without traversing in the opposite direction from equilibrium. It is interesting to note that in general, the application of a negative pulse (having the electrode at a higher potential with respect to the diaphragm) caused more deflection than application of a positive pulse with the same amplitude. This polarity dependant behavior could be caused by the charging characteristics of the dielectrics in the actuator structure. Positive and negative charges have different dissipation times in dielectrics and thus a polarity dependant behavior can be justified (Amjadi and Sessler 1997; Okusako et al. 1994; Wibbeler et al. 1998). Note that by increasing the pulse width, more contact time at high voltage was allowed for the diaphragm against the back-plate; however, the release time did not change by changing to pulse width above $60 \mu \mathrm{s}$. This suggests that the charging happened in a very short interval and stopped. The evidence suggests that charging mechanism was less efficient across the gap than between the $\mathrm{P}^{++}$silicon and the adjacent deposited dielectric. With the increase of the actuation voltage, as expected, the collapse time decreased due to the stronger electrostatic force engaged in the collapse. This change was significant for a positive pulse but negligible in the case of a negative pulse. The release time for all these cases was about $50 \mu$ s. Thus operation at higher voltages does not have an adverse effect on the release cycle and can be safely implemented to reduce the collapse time.

Table 3 shows the measured data for various designs. We used the same input signal for all designs in order to cancel the effect of input pulse amplitude and duration. Due to large variations in measurements or insufficient collapse of diaphragms, some of the data points were not available (N/A).

As mentioned in the discussion of the sensitivity of device dynamics to input voltage amplitude and duration, the limiting factor for increasing the operation frequency for design\#1 was the release time. This time showed a significant reduction from 50 to $16.5 \mu \mathrm{s}$ by changing the throat size from 15 to $35 \mu \mathrm{m}$. Design\#10 was the fastest among the measured devices in Table 3 and was capable of completing a full collapse-release cycle in $28 \mu \mathrm{s}$. This corresponds to operation frequency of $35.7 \mathrm{kHz}$. This design was able to provide full diaphragm deflection at this frequency.

It is also possible to use the interferometric measurements to decouple the structural and acoustic resonance points of the system and measure the latter directly. If the amplitude of motion of the diaphragm is kept constant while increasing the actuation frequency, the output acoustic power will be only a function of the acoustic behavior of the Helmholtz resonator. An experiment was performed to study this effect. In this experiment, the applied voltage at each frequency was modified in order to achieve six fringe movement for the diaphragm. This corresponded to changes in the input voltage in the range of 35.9-50.3 $\mathrm{V}$ (not monotonous). The collapse was avoided in order to reduce the introduction of non-linear effects. The acoustic output was measured with a microphone connected to a high-gain amplifier. Figure 6 shows the measured results. The microphone used for this experiment had been designed for audio frequencies and thus the measured values after $20 \mathrm{kHz}$ need to be corrected based on the gain-frequency curve of the system. Assuming that the roll-off happens at about $20 \mathrm{kHz}$, the peak at $20 \mathrm{kHz}$ cannot be interpreted as a definite acoustic output peak. On the other hand, if the microphone frequency response is flat (with very low gain) at higher frequencies, the second peak in the graph at $80 \mathrm{kHz}$ could be a credible one.

\subsection{Video particle imaging}

We used video particle imaging with $15 \mu \mathrm{m}$ silver-covered hollow glass particles to verify the occurrence of acoustic streaming. Although conductive particles were used in this experiment to avoid stiction to the surface, many of the particles that landed upon introduction on a non-vibrating surface, stuck and did not move during the entire experiment. Design\#1 resonators were actuated with a $40 \mathrm{kHz}$ sine wave $(80 \mathrm{kHz}$ diaphragm motion frequency) and consecutive video images, 1/30 s apart, were taken. The imaging was done at a frequency much lower than the oscillation frequency of the diaphragm due to limitations of our imaging system;

Table 3 Measured device performance for various designs

\begin{tabular}{|c|c|c|c|c|c|c|c|c|c|c|}
\hline Design\# & 1 & 2 & 3 & 4 & $5^{a}$ & 6 & 7 & 8 & 9 & 10 \\
\hline Collapse time $t_{c}(\mu \mathrm{s})$ & 10 & 44 & 26 & 24 & $\mathrm{~N} / \mathrm{A}$ & 14 & $\mathrm{~N} / \mathrm{A}$ & 14 & 15.5 & 11.5 \\
\hline Release time $t_{r}(\mu \mathrm{s})$ & 50 & 24 & 24 & 50 & $\mathrm{~N} / \mathrm{A}$ & $\mathrm{N} / \mathrm{A}$ & $\mathrm{N} / \mathrm{A}$ & 16 & 17 & 16.5 \\
\hline Ave Col./Rel. time $(\mu \mathrm{s})$ & 30 & 34 & 25 & 37 & $\mathrm{~N} / \mathrm{A}$ & $\mathrm{N} / \mathrm{A}$ & $\mathrm{N} / \mathrm{A}$ & 15 & 16.25 & 14 \\
\hline${ }^{b}$ Ave. exit velocity $(\mathrm{cm} / \mathrm{s})$ & 170.7 & 160.4 & 65.4 & 147.4 & $\mathrm{~N} / \mathrm{A}$ & $\mathrm{N} / \mathrm{A}$ & $\mathrm{N} / \mathrm{A}$ & 181.8 & 143.9 & 156.8 \\
\hline${ }^{c}$ Ave. exit flow $(\mathrm{cm} / \mathrm{s})$ & 50 & 45 & 47.5 & 53.3 & 85 & 45 & 40 & 24.2 & 48.3 & 54.2 \\
\hline
\end{tabular}

\footnotetext{
${ }^{a}$ Non-uniform hole design ${ }^{b}$ Calculated based on compressible laminar flow ${ }^{c}$ Measured with pulse drive at $1 \mathrm{kHz}$
} 


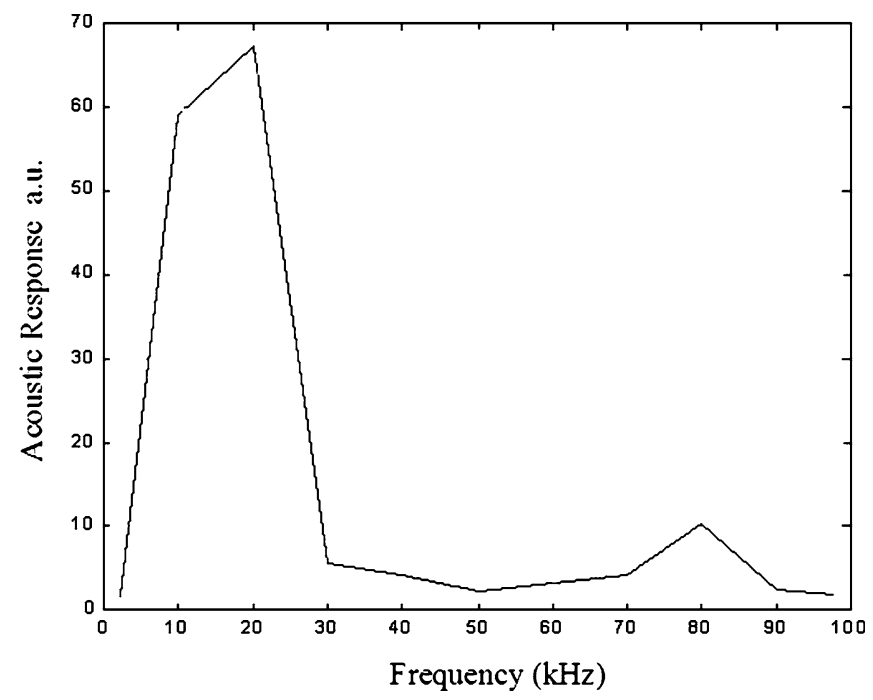

Fig. 6 Acoustic output versus diaphragm motion frequency. The diaphragm motion amplitude was kept constant at $1.46 \mu \mathrm{m}$ center deflection. The graph shows the location of the resonance points. In order to measure the ratio of the peak values, the graph should be compensated for the frequency response of the microphone

however, due to the small lateral velocity of the particles we were able to trace their trajectories in consecutive images. These trajectories clearly indicated the existence of a mean flow in $Y$ direction perpendicular to the throat exit, this in turn confirmed the occurrence of acoustic streaming. Figure 7 shows a typical sequence of images. The white arrow traces the location of a particle moving perpendicular to the throat due to streaming. The maximum particle velocity was estimated at $1.5 \mathrm{~mm} / \mathrm{s}$. It should be noted that the relative displacement of a particle with the above specifications in a flow field changing at $80 \mathrm{kHz}$ with the expected flow velocities is about $0.02 \mu \mathrm{m}$, a value much smaller than the $150 \mu \mathrm{m}$ traveling distance observed for the particles. This clearly verifies the occurrence of acoustic streaming and lateral mean flow over the surface of the device. Note that the particle velocity of $1.5 \mathrm{~mm} / \mathrm{s}$ does not directly correspond to the flow velocity due to the interaction of the particle and the substrate. We anticipate a significant reduction of the particle traveling velocity due to the Van Der Waals and electrostatic attraction forces between the surface and the particle.

\subsection{Hot-wire anemometer velocity measurements}

We used a $5 \mu \mathrm{m}$ wide hot-wire anemometer connected across a resistive bridge in order to measure the output jet velocity at the throat of the resonators. Readings were available in lower frequency operations $(\sim 1 \mathrm{kHz})$; however, with increase in operating frequency, a strong noise from the electrostatic actuator screened the sensor read-out. Due to this heavy noise effect, velocity measurement was not possible at resonance. In this section low frequency operation and in particular the pulse response of different designs are studied. Although all resonators were operated at $1 \mathrm{kHz}$, a frequency much lower than their resonance frequency, jet velocities close to $1 \mathrm{~m} / \mathrm{s}$ were measured.

Table 3 shows the average velocity measured for each design. The average collapse/release time is also presented in this table. Based on this and assumption of the laminar incompressible flow, the output jet velocity for each resonator was calculated. The calculated numbers are all larger than the measured values. This suggests that significant compressibility, and perhaps damping in the throat, happens in the device even during operation at low frequencies with pulse drive. The best outputs were measured from design\#5 with the non-uniform perforation design. This proved that the optimization for the perforation design had been accomplished and the response of the system had been improved significantly with respect to design\#1.

We measured the profile of the generated jets by tracing the hot-wire laterally (along the $Y$-direction in Fig. 1) and also perpendicular to the wafer surface (along $Z$-direction in Fig. 1). Figure 8 shows the measured lateral jet profile for a design\#5 device for various distances between the measurement point and the exit throat. The jet velocity decreased by $0.024 \mathrm{~dB} / \mu \mathrm{m}$ for distances less than $400 \mu \mathrm{m}$ from the exit throat.

\section{Conclusions}

Fabrication and characterization of high frequency Helmholtz resonators forming the unit cells for a distributed propulsion system are presented. We demonstrate a high yield $(>85 \%)$ fabrication process for fabrication of thousands of resonator units on a 4-in. wafer and in effect convert the wafer to a distributed propulsion system. A number of devices with various design parameters were fabricated using this microfabrication technique. The behavior of these devices was studied using methods ranging from laser interferometry to particle velocity imaging.

Electrostatic micro actuators provide the necessary displacement in the Helmholtz resonator cavities to generate the initial acoustic fields. By investigating various design parameters and full characterization of these high frequency actuators, we demonstrate how to improve their performance. A polarity dependant behavior in the operation of the electrostatic actuators was observed and associated with charging of the dielectrics in the actuator structure. The effect of actuator back-plate perforation design on diaphragm collapse and recovery times was studied. By matching the hole pattern to the collapse pattern of the diaphragm, significant improvement $(>70 \%)$ in device performance and output jet velocity was observed. The resonance points of the system were measured and it is established that they closely follow the theoretical predictions. Table 4 shows a range of parameters for 
Fig. 7 Particle trajectory imaging looking at the $X Y$ plane of a resonator. Picture sequences a-g were taken $1 / 30 \mathrm{~s}$ apart. Particles were introduced on the left side over the perforation area. They traveled more than $150 \mu \mathrm{m}$ and reached the throat on the right side. The white arrow follows one particle through the travel period
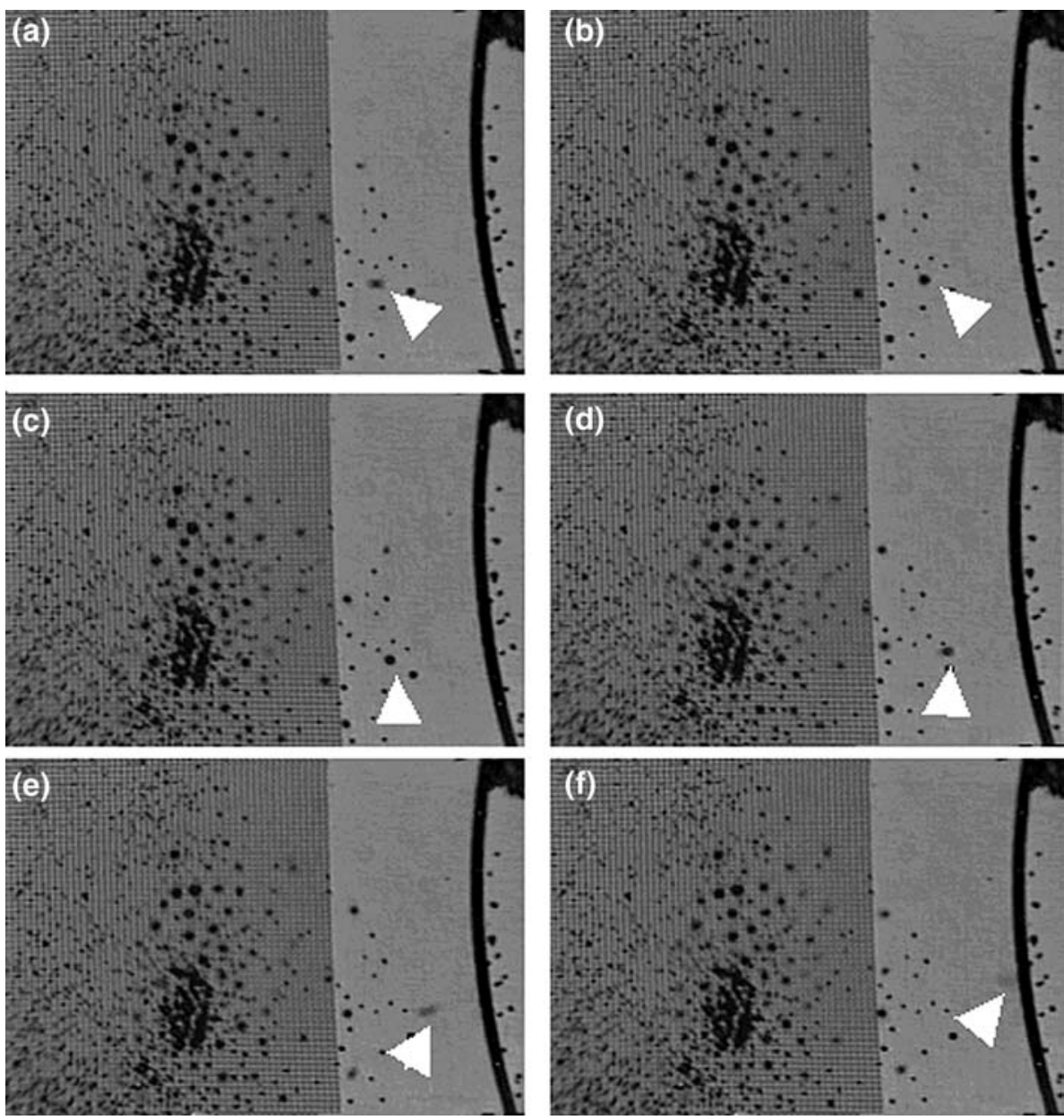

design\#1 as a representative device and the comparison between the theoretical calculations and the measured values. The occurrence of acoustic streaming in the

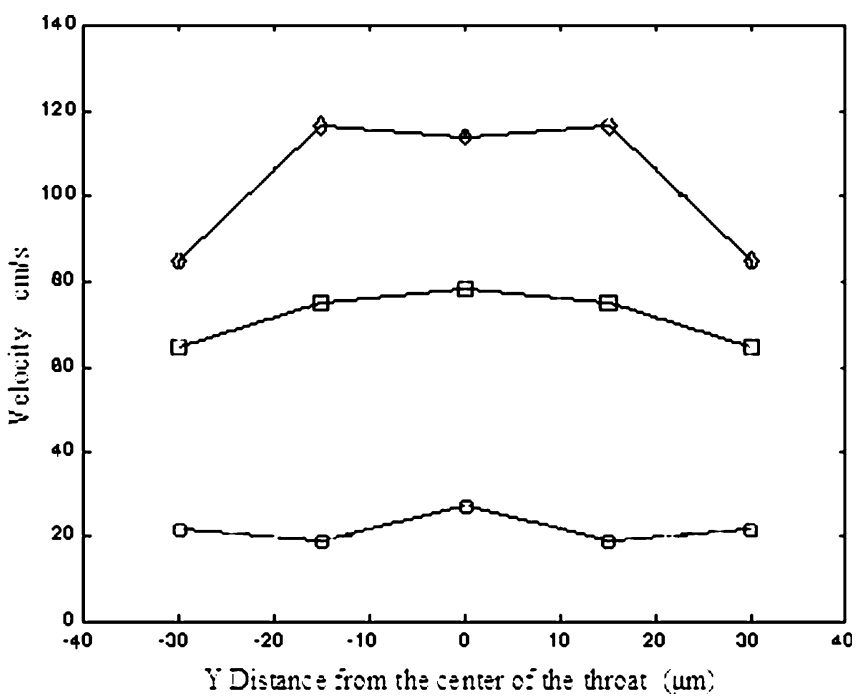

Fig. 8 Measured lateral jet profile. For each series of data points, $Z$ value (see Fig. 1) of the hot-wire anemometer was fixed and its location along the $Y$ direction was varied over the throat. Diamond: $64 \mu \mathrm{m}$ from the throat, Square: $220 \mu \mathrm{m}$ from the throat and Circle: $380 \mu \mathrm{m}$ away from the throat micro scale was verified by particle velocity imaging. A pulse drive was used to generate jet streams from a design\#5 device and the output jet was profiled both along and away from the centerline. These measurements were performed at $1 \mathrm{kHz}$ drive signal frequency due to the small signal to noise ratio of the anemometer at higher frequencies. The highest jet velocity measured at low frequency (pulse at $1 \mathrm{kHz}$ ) off-resonance operation was above $1 \mathrm{~m} / \mathrm{s}$. The measured velocities with pulse drive were lower than the calculated values. The calculated values should be modified by inclusion of compressibility effects in pulse operation and by compensation for the hot-wire frequency response (roll off at $7 \mathrm{kHz}$ ) in order to get a closer approximation to the measured values.

In conclusion, we demonstrate that acoustic streaming at the exit of a Helmholtz resonator can create micron-scale jet streams and that an array of these jets can be microfabricated. We can predict performance parameters of these jets by using equations derived in (Parviz et al. 2005). We propose that these jets can be used in a number of applications such as in flow vectoring, chip cooling, and in pumping and mixing in micro chemical reactors. Mainly, we propose these jets as a candidate to form a distributed micro propulsion system for micro flying vehicles. 
Table 4 Comparison of calculated and measured parameters for Design\#1

\begin{tabular}{lll}
\hline Parameter & Calculated & Measured \\
\hline DC Diaphragm collapse voltage & $52.5 \mathrm{~V}$ & $60 \mathrm{~V}$ \\
Helmholtz resonance frequency & $59.1 \mathrm{kHz}$ & $80 \mathrm{kHz}$ \\
First resonance point of the device & $50 \mathrm{kHz}$ & $52 \mathrm{kHz}$ \\
Second resonance point of the device & $175 \mathrm{kHz}$ & $190 \mathrm{kHz}$ \\
Occurrence of acoustic streaming & Yes & Yes \\
Pulse drive jet velocity at 1 kHz & $170.7 \mathrm{~cm} / \mathrm{s}$ & $50 \mathrm{~cm} / \mathrm{s}$ \\
\hline
\end{tabular}

Acknowledgments The authors would like to thank Dr. Allen Chou and Dr. Chunbo Zhang for their help with the microfabrication of the resonators. They also would like to thank Christopher Morris for his help in preparing the manuscript. Funding for this work was provided by the Defense Advanced Research Projects Agency (DARPA) through contract\#N00019-98-K-0111.

\section{References}

Amjadi H, Sessler GM (1997) Charge storage in APCVD silicon nitride. In: Proceedings of the IEEE conference on electrical insulation and dielectric phenomena. Minneapolis pp 64-67

Coe DJ et al (1994) Micromachined jets for manipulation of macro flows. In: Solid-state sensor and actuator workshop digest. Hilton Head, SC, pp 243-247
Coe DJ et al (1995) Addressable micromachined jet arrays. In: Proceedings of the transducers '95. Stockholm, Sweden, pp 329-332

Muller MO et al (2000) Thrust performance of micromachined synthetic jets. In: AIAA fluids 2000 conference. Denver, Colorado, pp 2000-2404

Okusako T et al (1994) Dissipation of contact electrified electrons on dielectric thin films with silicon substrate. Jpn J App 1 Phys 33:L959-L961

Parviz BA et al (2000) A wafer integrated array of micromachined electrostatically driven ultrasonic resonators for microfluidic app lications. In: Proceedings of the 13th IEEE MEMS international conference. Miyazaki, Japan, pp 34-39

Parviz BA et al (2001) Performance of ultrasonic electrostatic resonators for use in micro propulsion. In: Proceedings of the 14th IEEE MEMS international conference. Interlaken, Switzerland, pp 586-589

Parviz BA, Najafi K, Muller MO, Bernal LP, Washabaugh PD (2005) Electrostatically driven synthetic microjet arrays as a propulsion method for micro flight. Part I: principles of operation, modeling, and simulation. Microsyst Technol DOI 10.1007/s00542-005-0599-0

Wibbeler J et al (1998) Parasitic charging of dielectric surfaces in capacitive microelectromechanical systems. Sens Actuators A71:74-80

Wolffenbuttel RF, Wise KD (1994) Low-temperature silicon waferto-wafer bonding using gold at eutectic temperature. Sens Actuators A43:223-229 